\title{
Diagnostic value of preoperative inflammatory markers in patients with glioma: a multicenter cohort study
}

\author{
*Shi-hao Zheng, MD,' Jin-lan Huang, PhD, ${ }^{2,4}$ Ming Chen, MD, ${ }^{3}$ Bing-long Wang, BS, ${ }^{2}$ \\ Qi-shui Ou, PhD, ${ }^{2}$ and Sheng-yue Huang, BS ${ }^{1}$
}

\begin{abstract}
'Department of Neurosurgery, Fujian Provincial Hospital; '2Department of Clinical Laboratory, First Affiliated Hospital of Fujian Medical University, Fuzhou, Fujian; ${ }^{3}$ Department of Neurosurgery, Xin Hua Hospital, Affiliated with Shanghai Jiao Tong University School of Medicine, Shanghai; and ${ }^{4}$ Laboratory Medicine Center, Nanfang Hospital, Southern Medical University, Guangzhou, Guangdong, People's Republic of China
\end{abstract}

OBJECTIVE Glioma is the most common form of brain tumor and has high lethality. The authors of this study aimed to elucidate the efficiency of preoperative inflammatory markers, including neutrophil/lymphocyte ratio (NLR), derived NLR (dNLR), platelet/lymphocyte ratio (PLR), lymphocyte/monocyte ratio (LMR), and prognostic nutritional index (PNI), and their paired combinations as tools for the preoperative diagnosis of glioma, with particular interest in its most aggressive form, glioblastoma (GBM).

METHODS The medical records of patients newly diagnosed with glioma, acoustic neuroma, meningioma, or nonlesional epilepsy at 3 hospitals between January 2011 and February 2016 were collected and retrospectively analyzed. The values of NLR, dNLR, PLR, LMR, and PNI were compared among patients suffering from glioma, acoustic neuroma, meningioma, and nonlesional epilepsy and healthy controls by using nonparametric tests. Correlations between NLR, $\mathrm{dNLR}, \mathrm{PLR}, \mathrm{LMR}, \mathrm{PNI}$, and tumor grade were analyzed. Receiver operating characteristic (ROC) curve analysis was performed to evaluate the diagnostic significance of NLR, dNLR, PLR, LMR, PNI, and their paired combinations for glioma, particularly GBM.

RESULTS A total of 750 patients with glioma (Grade I, 81 patients; Grade II, 208 patients; Grade III, 169 patients; Grade IV [GBM], 292 patients), 44 with acoustic neuroma, 271 with meningioma, 102 with nonlesional epilepsy, and 682 healthy controls were included in this study. Compared with healthy controls and patients with acoustic neuroma, meningioma, or nonlesional epilepsy, the patients with glioma had higher values of preoperative NLR and dNLR as well as lower values of LMR and PNI, whereas PLR was higher in glioma patients than in healthy controls and patients with nonlesional epilepsy. Subgroup analysis revealed a positive correlation between NLR, dNLR, PLR, and tumor grade but a negative correlation between LMR, PNI, and tumor grade in glioma. For glioma diagnosis, the area under the curve (AUC) obtained from the ROC curve was 0.722 (0.697-0.747) for NLR, $0.696(0.670-0.722)$ for dNLR, $0.576(0.549-0.604)$ for PLR, 0.760 (0.738-0.783) for LMR, and $0.672(0.646-0.698)$ for PNI. The best diagnostic performance was obtained with the combination of NLR+LMR and dNLR+LMR, with AUCs of 0.777 and 0.778 , respectively. Additionally, NLR (AUC $0.860,95 \% \mathrm{Cl} 0.832-0.887)$, dNLR $(0.840,0.810-0.869)$, PLR $(0.678,0.641-0.715)$, LMR $(0.837,0.811-0.863)$, and PNI $(0.740,0.706-0.773)$ had significant predictive value for GBM compared with healthy controls and other disease groups. As compared with the Grade I-III glioma patients, the GBM patients had an AUC of $0.811(95 \% \mathrm{Cl} 0.778-0.844)$ for NLR, 0.797 (0.763-0.832) for dNLR, 0.662 (0.622-0.702) for PLR, $0.743(0.707-0.779)$ for LMR, and 0.661(0.622-0.701) for PNI. For the paired combinations, NLR+LMR demonstrated the highest accuracy.

CONCLUSIONS The NLR+LMR combination was revealed as a noninvasive biomarker with relatively high sensitivity and specificity for glioma diagnosis, the differential diagnosis of glioma from acoustic neuroma and meningioma, GBM diagnosis, and the differential diagnosis of GBM from low-grade glioma.

https://thejns.org/doi/abs/10.3171/2017.3.JNS161648

KEY WORDS glioma; glioblastoma; biomarkers; neutrophil lymphocyte ratio; lymphocyte monocyte ratio; oncology

ABBREVIATIONS AUC = area under the curve; $\mathrm{dNLR}=$ derived NLR; GBM = glioblastoma; IL-10 = interleukin 10; LMR = lymphocyte/monocyte ratio; NLR = neutrophil/ lymphocyte ratio; $\mathrm{PLR}=$ platelet/lymphocyte ratio; $\mathrm{PNI}=$ prognostic nutritional index; $\mathrm{ROC}=$ receiver operating characteristic; TGF $-\beta=$ tumor growth factor $-\beta ;$ WBC $=$ white blood cell.

SUBMITTED June 27, 2016. ACCEPTED March 17, 2017

INCLUDE WHEN CITING Published online November 3, 2017; DOI: 10.3171/2017.3.JNS161648.

* Drs. Zheng, J. I. Huang, and Chen contributed equally to this work. 
$\mathrm{G}$ LIOMA is the most frequent primary brain tumor, accounting for $81 \%$ of malignant brain and central nervous system tumors. ${ }^{22}$ The most aggressive and common type of glioma is glioblastoma (GBM; WHO Grade IV), with an annual incidence of approximately 3.5 cases in 100,000 people per year. ${ }^{13}$ Despite the therapeutic advances made in the past few decades, such as resection, irradiation with adjuvant temozolomide, and experimental chemotherapy, the overall 5-year survival rate of glioma patients remains poor. ${ }^{22,28}$ In particular, GBM patients have the poorest overall survival, with a median survival of only 12-15 months. ${ }^{1}$ Currently, histological examination of tumor tissue obtained at biopsy or resection serves as the standard procedure for definitive diagnosis of glioma, whereas CT and MRI are supplemental procedures for disease staging and treatment response monitoring. ${ }^{1,21}$ However, both the histopathological and neuroimaging tests are insensitive and expensive and can cause hemorrhage or neurological damage. ${ }^{23}$ Moreover, unlike other cancers such as prostate cancer, glioma has no sensitive or specific serum marker that has been clinically applied for its detection, disease staging, treatment response monitoring, and prognosis. ${ }^{17,29}$ Thus, there is an urgent need for minimally invasive biomarkers with improved sensitivity and specificity and lower costs for glioma, especially GBM.

Emerging studies have highlighted the crucial role of the host inflammatory response in cancer progression and prognosis. ${ }^{19}$ Generally, the inflammatory response is characterized by changes in the levels of white blood cells (WBCs), neutrophils, lymphocytes, monocytes, platelets, C-reactive protein, and albumin. ${ }^{5,6}$ These blood parameters are simple, low cost, and easy to measure with widely used and standardized assays. Various combinations of these factors have been measured to derive inflammation-based scores, including neutrophil/lymphocyte ratio (NLR), derived NLR (dNLR), platelet/lymphocyte ratio (PLR), lymphocyte/monocyte ratio (LMR), and prognostic nutritional index (PNI). Recently, all 5 of these markers have been proven to refine stratification of patients to treatment and predict survival in various cancers. ${ }^{5,9,24}$ As regards glioma, an increase in preoperative NLR has been linked to poorer survival in GBM patients enrolled in 2 cohort studies. ${ }^{3,12}$ Moreover, McNamara et al. have reported that an NLR > 4 prior to a second surgery is a poor prognostic factor in GBM. ${ }^{20}$ However, previous studies have focused mainly on the value of these markers in predicting patient survival and estimating GBM prognosis. The diagnostic value of these 5 markers in glioma as well as GBM has not been elucidated. Aims of the present study were to describe differences in the levels of several preoperative inflammatory markers among patients with glioma; patients with other intracranial diseases such as acoustic neuroma, meningioma, and nonlesional epilepsy; and healthy controls and to assess the utility of NLR, dNLR, PLR, LMR, PNI, and their combinations as tools for the preoperative diagnosis of glioma with a particular interest on GBM diagnosis.

\section{Methods}

\section{Patients and Healthy Controls}

The medical records of patients newly diagnosed with glioma, acoustic neuroma, meningioma, or nonlesional epilepsy at 3 hospitals (Fujian Provincial Hospital, Guangzhou Nangfang Hospital, Shanghai Xin Hua Hospital) between January 2011 and February 2016 were collected and retrospectively analyzed. All patients included in the analysis had to meet the following criteria: 1) glioma and tumor grading histologically verified in a resection or biopsy specimen according to WHO criteria, acoustic neuroma and meningioma histologically proven in a resection, or nonlesional epilepsy verified according to the practical clinical definition of epilepsy developed by the International League Against Epilepsy (ILAE) in 2014; 2) no prior cancer-specific pretreatment, such as chemotherapy or radiotherapy; 3) no hematological disease, current infectious disease, hyperpyrexia, diabetes mellitus, metabolic syndrome, serious heart disease, hypertension, severe renal or hepatic dysfunction, other cancer, autoimmune disease, inflammatory disease, or medication usage related to an inflammatory condition; 4) complete data on preoperative blood count and serum albumin levels; and 5) informed consent. Ultimately, 750 patients with glioma, 44 with acoustic neuroma, 271 with meningioma, and 102 with nonlesional epilepsy were included in the analysis. For the control group, we reviewed the records of 682 ageand sex-matched healthy individuals who had undergone their annual health check at the hospital. This study was approved by the institutional ethics committee.

\section{Data Collection}

Demographic and clinicopathological variables, including age, sex, tumor grade, histological type, and so forth, were retrieved from the patient medical records. Within 1 week of surgery, blood samples were routinely taken for a blood test and hepatic function test as part of the standard preoperative workup. All tests were performed by staff in the clinical laboratory department within 2 hours of collection. The WBC, neutrophil, lymphocyte, monocyte, and platelet counts were collected from the routine blood test, and albumin levels were collected from the hepatic function test. Moreover, preoperative NLR (quotient of neutrophil count to lymphocyte count), dNLR (quotient of [WBC count - neutrophil count] to lymphocyte count), PLR (ratio of platelet count to lymphocyte count), LMR (ratio of lymphocyte count to monocyte count), and PNI (albumin count $[\mathrm{g} / \mathrm{L}]+$ total lymphocyte count $\times 5$ ) were calculated .

\section{Statistical Analysis}

Statistical analysis was performed using SPSS version 13.0 (SPSS Inc.). Initially, normal distribution of the variables was analyzed using the Kolmogorov-Smirnov test. All the data in our study were not subject to normal distribution and were presented as median with range. For nonparametric data, the Mann-Whitney U-test was used for comparisons between groups. The association of NLR, dNLR, PLR, LMR, and PNI with tumor grade was evaluated using the Kruskal-Wallis test followed by a post hoc test and a multivariate regression model. Correlation was judged by the Pearson correlation coefficient test for 2 variables with normal distribution or by the Spearman correlation coefficient test for abnormally distributed vari- 
TABLE 1. Preoperative characteristics of patients with glioma, meningioma, acoustic neuroma, or nonlesional epilepsy and healthy controls

\begin{tabular}{|c|c|c|c|c|c|}
\hline Parameter & Healthy Controls & Nonlesional Epilepsy & Acoustic Neuroma & Meningioma & Glioma \\
\hline Age in yrs & $42(6-84)$ & $41.5(9-88)$ & $48.5(25-75)^{*}$ & $52(18-84)^{*} \dagger \ddagger$ & $43(1-85) \ddagger \S$ \\
\hline No. of patients & 682 & 102 & 44 & $271^{*} \dagger \ddagger$ & $750 \S$ \\
\hline Male & 380 & 54 & 20 & 78 & 416 \\
\hline Female & 302 & 48 & 24 & 193 & 334 \\
\hline WBCs in $10^{9} / \mathrm{L}$ & $6.11(3.58-9.47)$ & $5.78(3.10-10.77)$ & $5.70(2.40-10.95)^{*}$ & $5.90(2.63-16.42)$ & $7.20(2.72-25.59)^{\star} \dagger \ddagger \S$ \\
\hline Neutrophils in $10^{9} / \mathrm{L}$ & $3.26(1.80-6.09)$ & $3.24(1.38-6.39)$ & $2.91(1.20-7.52)^{*}$ & $3.4(1.21-14.20) \dagger \ddagger$ & $4.375(0.41-20.90)^{*} \dagger \ddagger \S$ \\
\hline Lymphocytes in $109 / \mathrm{L}$ & $2.22(1.2-3.8)$ & $1.99(1.0-4.9)^{*}$ & $1.95(0.82-3.0)^{*}$ & $1.8(0.7-4.5)^{*} \dagger$ & $1.90(0.4-5.2)^{*} \mp \S$ \\
\hline Monocytes in $10^{\circ} / \mathrm{L}$ & $0.36(0.13-0.77)$ & $0.34(0.14-0.71)$ & $0.32(0.19-0.57)^{*}$ & $0.35(0.08-1.07)$ & $0.46(0.06-2.72)^{\star} \dagger \pm \S$ \\
\hline Platelets in $10^{9} / \mathrm{L}$ & $242(130-355)$ & $230(97-395)^{*}$ & $226(76-341)$ & $233(94-521)^{\star}$ & $234(46-573)$ \\
\hline Albumin in $\mathrm{g} / \mathrm{L}$ & $43.1(35.0-54.5)$ & $44.1(34.0-51.1)$ & $44.1(38.1-57)^{*}$ & $43(31.4-52) \dagger \ddagger$ & $40.55(23.5-54.0)^{*} \dagger \neq \S$ \\
\hline NLR & $1.49(0.73-3.00)$ & $1.55(0.69-3.67)$ & $1.60(0.80-2.81)$ & $1.82(0.71-10.14)^{*} \dagger \ddagger$ & $2.25(0.19-22.47)^{*} \dagger \neq \S$ \\
\hline $\mathrm{dNLR}$ & $1.21(0.60-2.42)$ & $1.21(0.56-2.30)$ & $1.20(0.68-2.37)$ & $1.38(0.56-8.33)^{\star} \dagger \ddagger$ & $1.65(0.03-10.73)^{*} \dagger \ddagger \S$ \\
\hline PLR & $108.60(46.10-241.27)$ & $110.13(36.73-258.17)$ & $112.35(52.96-367.07)$ & $127.00(41.11-347.25)^{*} \dagger$ & $123.92(19.67-482.46)^{*} \dagger$ \\
\hline LMR & $6.18(3.51-13.16)$ & $5.93(1.75-13.83)$ & $6.08(2.98-14.55)$ & $5.28(1.32-16.38)^{*} \dagger$ & $4.09(0.52-16.43)^{\star} \dagger \pm \S$ \\
\hline $\mathrm{PNI}$ & $54.33(44.60-67.85)$ & $54.78(43.00-65.75)$ & $54.50(43.60-67.00)$ & $52.40(39.50-70.25)^{*} \dagger \ddagger$ & $50.68(29.45-72.50)^{*} \dagger \pm \S$ \\
\hline
\end{tabular}

Values are median (range) unless otherwise indicated.

* $p<0.05$, compared to healthy controls.

$\dagger p<0.05$, compared to patients with nonlesional epilepsy.

$\ddagger p<0.05$, compared to patients with acoustic neuroma.

$\S p<0.05$, compared to patients with meningioma.

ables. The diagnostic performance of NLR, dNLR, PLR, LMR, PNI, and their combinations was assessed by values of the area under the curve (AUC) obtained from the receiver operating characteristic (ROC) curve. A 2-tailed $p$ value $<0.05$ was considered statistically significant.

\section{Results \\ Study Population}

A total of 750 patients with glioma, 44 with acoustic neuroma, 271 with meningioma, and 102 with nonlesional epilepsy and a control group of 682 healthy participants were included in the final analysis. Detailed demographic information for the study subjects are presented in Table 1. The glioma patients ranged in age from 1 to 85 years, and their median age was 43 years. The glioma cohort consisted of 416 men (55.47\%) and 334 women (44.53\%). The median age (range) for patients with nonlesional epilepsy, acoustic neuroma, and meningioma was 41.5 years (9-88 years), 48.5 (25-75), and 52 (18-84), respectively. For healthy controls, the median age was 42 years (range 6-84 years) and 380 of them $(55.72 \%)$ were male. At the time of diagnosis, lesions were classified as Grade I in 81 patients $(10.8 \%)$, Grade II in $208(27.73 \%)$, Grade III in 169 (22.53\%), and Grade IV (GBM) in 292 (38.93\%), according to the 2007 WHO criteria.

\section{Comparison of Preoperative Inflammatory Markers}

As shown in Table 1, significantly higher preoperative absolute WBC, neutrophil, and monocyte counts were observed in the glioma patients than in the nonlesional epilepsy, acoustic neuroma, and meningioma patients and healthy controls, whereas albumin levels in the glioma patients were markedly lower than those in the other groups. A significant decrease in absolute lymphocyte counts was observed between glioma patients and all other groups except for the patients with nonlesional epilepsy. Surprisingly, there was no significant difference in platelet counts between these groups. As for the calculated laboratory parameters, NLR, dNLR, and PLR were elevated in glioma patients relative to healthy controls (Fig. 1). However, much lower median values of LMR and PNI were noted in the glioma group. Interestingly, higher levels of NLR and dNLR, as well as lower levels of LMR and PNI, were observed in glioma patients than in the patients with other intracranial diseases.

\section{Correlation of Preoperative Inflammatory Markers With Glioma Grade}

We further investigated NLR, dNLR, PLR, LMR, and PNI according to glioma grade. A Kruskal-Wallis test revealed statistically significant interactions between tumor grade (I-IV) and NLR ( $p<0.001)$, dNLR $(\mathrm{p}<0.001)$, PLR ( $<<0.001)$, and LMR ( $<<0.001)$, PNI $(\mathrm{p}<0.001)$, respectively. Moreover, positive correlations were observed between NLR $(r=0.550, p<0.001)$, dNLR $(r=0.526, p$ $<0.001)$, PLR $(r=0.286, p<0.001)$, and glioma grade but negative correlations between LMR $(r=-0.426$, $\mathrm{p}<$ 0.001 ), PNI ( $\mathrm{r}=-0.318, \mathrm{p}<0.001$ ), and tumor grade (Fig. 2). Post hoc testing indicated a different pattern of changes for these 5 markers. More specifically, an increasing trend was observed for NLR and dNLR, with median NLR values of 1.56 for Grade I gliomas, 1.79 for Grade II, 2.19 for Grade III, and 3.61 for Grade IV (GBM) and median 

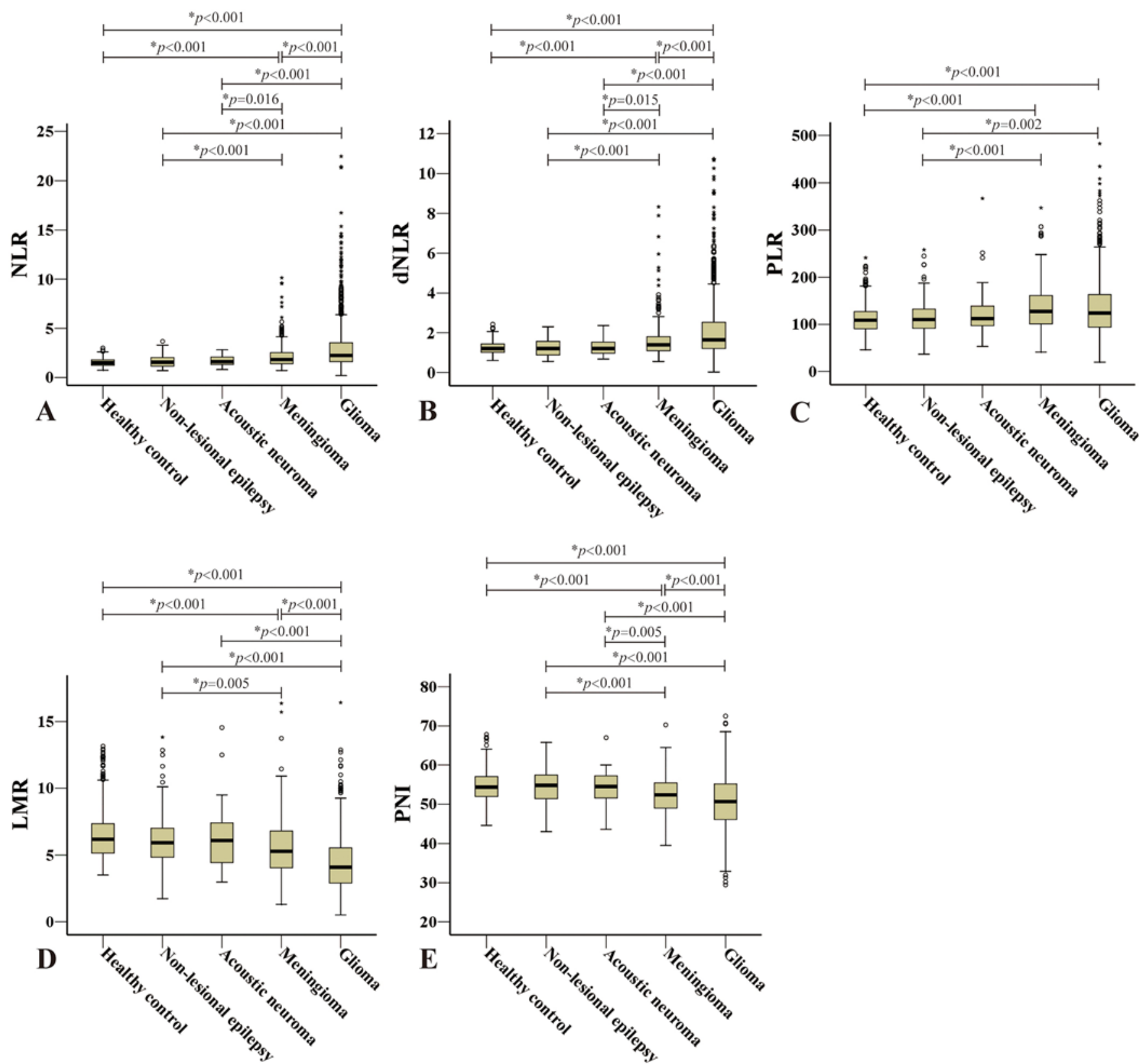

FIG. 1. Box plots of preoperative NLR (A), dNLR (B), PLR (C), LMR (D), and PNI (E) in patients with glioma, nonlesional epilepsy, acoustic neuroma, and meningioma and healthy controls. The boxes and whiskers represent interquartile range and full range, respectively. The data points past the whiskers indicate outliers. Figure is available in color online only.

dNLR values of 1.24 for Grade I, 1.34 for Grade II, 1.60 for Grade III, and 2.53 for Grade IV (Fig. 2A and B and Table 2). A similar trend was observed for PLR (Fig. 2C), and patients with an advanced grade glioma (III and IV) had higher NLR, dNLR, and PLR values relative to those in patients with low-grade glioma (I and II). In contrast, the level of LMR decreased in patients with Grade III gliomas, and a marked decrease was noted in patients with Grade IV gliomas (GBM; Fig. 2D). Additionally, PNI decreased as the tumor grade increased, with median values of 55.00 for Grade I, 51.50 for Grade II, 50.80 for Grade III, and 48.45 for GBM (Fig. 2E). Intriguingly, the highest values of NLR, dNLR, and PLR, as well as the low- est values of LMR and PNI, were noted in patients with GBM. In comparison with the healthy controls, the only significant variation in Grade I gliomas was detected at the LMR level.

\section{Evaluation of Diagnostic Efficacy of Preoperative Inflammatory Markers and Their Combinations in Patients With Glioma}

Figure 3 shows the diagnostic value (ROC curves) of NLR, dNLR, PLR, LMR, PNI, and their paired combinations for glioma patients. The corresponding AUC values appear in Table 3. The AUC was $0.722(0.697-0.747)$ for 

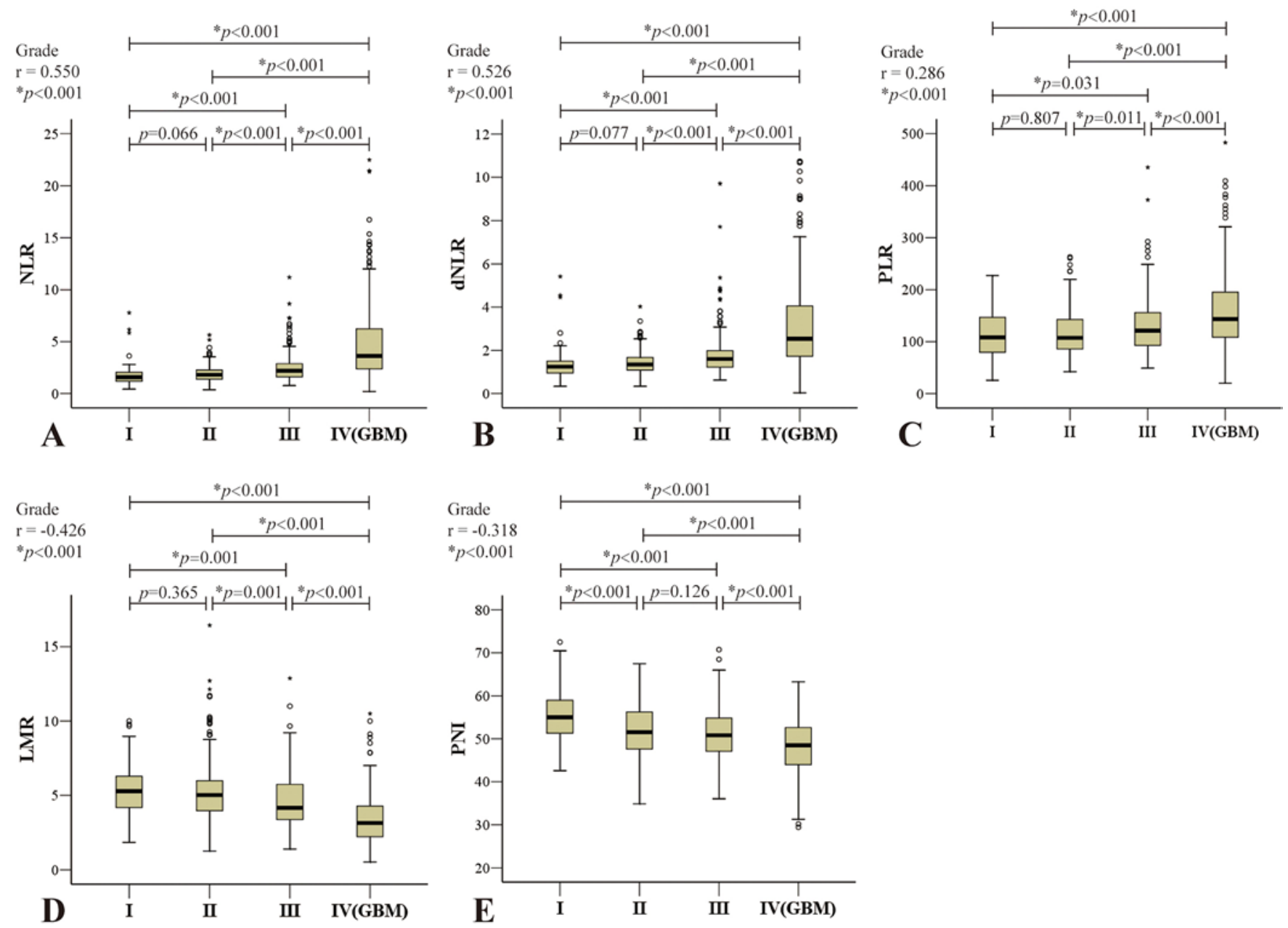

FIG. 2. Box plots of preoperative NLR (A), dNLR (B), PLR (C), LMR (D), and PNI (E) compared with glioma grade. The boxes and whiskers represent interquartile range and full range, respectively. The data points past the whiskers indicate outliers. Figure is available in color online only.

NLR, $0.696(0.670-0.722)$ for dNLR, $0.576(0.549-0.604)$ for PLR, 0.760 (0.738-0.783) for LMR, and $0.672(0.646-$ 0.698) for PNI when patients with glioma were tested against healthy controls plus patients with nonlesional epilepsy, acoustic neuroma, and meningioma. Although significant correlations among these 5 inflammatory markers were observed in glioma (Fig. 3A), LMR demonstrated the highest accuracy in predicting glioma (Fig. 3B). We also evaluated paired combinations of these markers for the diagnosis of glioma. The best diagnostic value was obtained with the combination of NLR+LMR and $\mathrm{dNLR}+\mathrm{LMR}$, with an AUC of 0.777 and 0.778 , respectively (Fig. 3C).

Given the fact that the highest values of NLR, dNLR, and PLR, as well as the lowest values of LMR and PNI, were all noted in patients with GBM, we assessed the diagnostic value of each of these markers and their paired combinations in patients with GBM compared with healthy controls plus patients with nonlesional epilepsy, acoustic neuroma, and meningioma and compared with patients with glioma Grades I-III. As shown in Fig. 4A and Table 3 , an ROC analysis indicated that NLR (AUC 0.860, 95\% CI 0.832-0.887), dNLR (0.840, 0.810-0.869), PLR (0.678, $0.641-0.715)$, LMR $(0.837,0.811-0.863)$, and PNI (0.740,
0.706-0.773) had significant predictive value for GBM as compared with controls and the other disease groups. When patients with GBM were tested against those with glioma Grades I-III, the AUC was 0.811 (95\% CI $0.778-$ $0.844)$ for NLR, 0.797 (0.763-0.832) for dNLR, 0.662 (0.622-0.702) for PLR, 0.743 (0.707-0.779) for LMR, and 0.661 (0.622-0.701) for PNI (Fig. 4B). Among all 5 of these markers, NLR had the best accuracy for GBM diagnosis and may be a discriminative parameter in predicting the grade of glioma. For paired combinations, NLR+LMR demonstrated the highest accuracy (Fig. 4C and D).

\section{Discussion}

Recent studies have considered glioma, especially GBM, to be a type of cancer closely correlated to chronic inflammation..$^{25}$ Recently, inflammatory markers have been reported to be useful diagnostic factors for various cancers. For instance, preoperative NLR and PLR have been identified as potential biomarkers for early detection, disease staging, and monitoring of colorectal cancer. ${ }^{14,15}$ However, little is known about the diagnostic value of inflammatory markers in patients with glioma including 
TABLE 2. Correlations between preoperative inflammatory markers and glioma grade

\begin{tabular}{|c|c|c|c|c|c|}
\hline \multirow[b]{2}{*}{ Marker } & \multirow[b]{2}{*}{ Healthy Controls } & \multicolumn{4}{|c|}{ Glioma Grade } \\
\hline & & $I(n=81)$ & II (n= 208) & III $(n=169)$ & IV (GBM; n = 292) \\
\hline WBCs in $10^{9} / \mathrm{L}$ & $6.11(3.58-9.47)$ & $7.05(4.30-25.59)^{\star}$ & $6.35(2.72-16.40)^{*} \dagger$ & $6.84(3.10-19.06)^{\star} \ddagger$ & $8.80(3.00-24.50)^{*} \dagger \ddagger \S$ \\
\hline $\begin{array}{l}\text { Neutrophils in } \\
10^{9} / \mathrm{L}\end{array}$ & $3.26(1.80-6.09)$ & $3.89(2.02-20.90)^{*}$ & $3.60(0.99-9.93)^{*}$ & $4.10(1.84-15.10)^{\star} \ddagger$ & $6.15(0.41-20.20)^{*} \dagger \ddagger \S$ \\
\hline $\begin{array}{l}\text { Lymphocytes in } \\
10 \% / \mathrm{L}\end{array}$ & $2.22(1.2-3.8)$ & $2.40(1.2-5.2)^{*}$ & $2.03(0.9-4.5)^{*} \dagger$ & $1.97(0.5-4.2)^{\star} \dagger$ & $1.60(0.4-4.6)^{\star} \dagger \ddagger \S$ \\
\hline $\begin{array}{l}\text { Monocytes in } \\
10 \% / \mathrm{L}\end{array}$ & $0.36(0.13-0.77)$ & $0.47(0.21-2.72)^{*}$ & $0.42(0.14-1.48)^{*} \dagger$ & $0.47(0.06-1.67)^{\star} \ddagger$ & $0.51(0.14-2.15)^{\star} \mp \S$ \\
\hline $\begin{array}{l}\text { Platelets in } \\
10 \% / \mathrm{L}\end{array}$ & $242(130-355)$ & $258(118-502)^{*}$ & $229.5(71-526)^{\star} \dagger$ & $238(61-573) \dagger$ & $230.5(46-514) \dagger$ \\
\hline Albumin in $\mathrm{g} / \mathrm{L}$ & $43.1(35.0-54.5)$ & $42.0(33.5-53.0)^{*}$ & $40.7(27.0-54.0)^{*} \dagger$ & $40.9(25.8-54.0)^{*}$ & $39.55(23.50-53.0)^{*} \dagger \neq \S$ \\
\hline NLR & $1.49(0.73-3.00)$ & $1.56(0.42-7.78)$ & $1.79(0.37-5.64)^{*}$ & $2.19(0.76-11.20)^{*} \dagger \ddagger$ & $3.61(0.19-22.47)^{*} \dagger \neq \S$ \\
\hline $\mathrm{dNLR}$ & $1.21(0.60-2.42)$ & $1.24(0.33-5.41)$ & $1.34(0.33-4.03)^{*}$ & $1.60(0.62-9.71)^{*} \dagger \ddagger$ & $2.53(0.03-10.73)^{*} \dagger \ddagger \S$ \\
\hline PLR & $108.60(46.10-241.27)$ & $108.10(25.82-227.15)$ & $107.47(41.93-263.00)$ & $121.29(48.83-434.78)^{\star} \dagger \ddagger$ & $143.21(19.67-482.46)^{\star} \dagger \neq \S$ \\
\hline LMR & $6.18(3.51-13.16)$ & $5.28(1.84-10.00)^{*}$ & $5.02(1.25-16.43)^{\star}$ & $4.15(1.39-12.88)^{*} \dagger \ddagger$ & $3.15(0.52-10.50)^{*} \dagger \neq \S$ \\
\hline PNI & $54.33(44.60-67.85)$ & $55.00(42.60-72.50)$ & $51.50(34.85-67.50)^{*} \dagger$ & $50.80(36.05-70.75)^{*} \dagger$ & $48.45(29.45-63.25)^{\star} \dagger \ddagger \S$ \\
\hline
\end{tabular}

All values are median (range).

${ }^{*} p<0.05$, compared to healthy controls.

$\dagger p<0.05$, compared to Grade I glioma patients.

$\ddagger p<0.05$, compared to Grade II glioma patients.

$\S p<0.05$, compared to Grade III glioma patients.
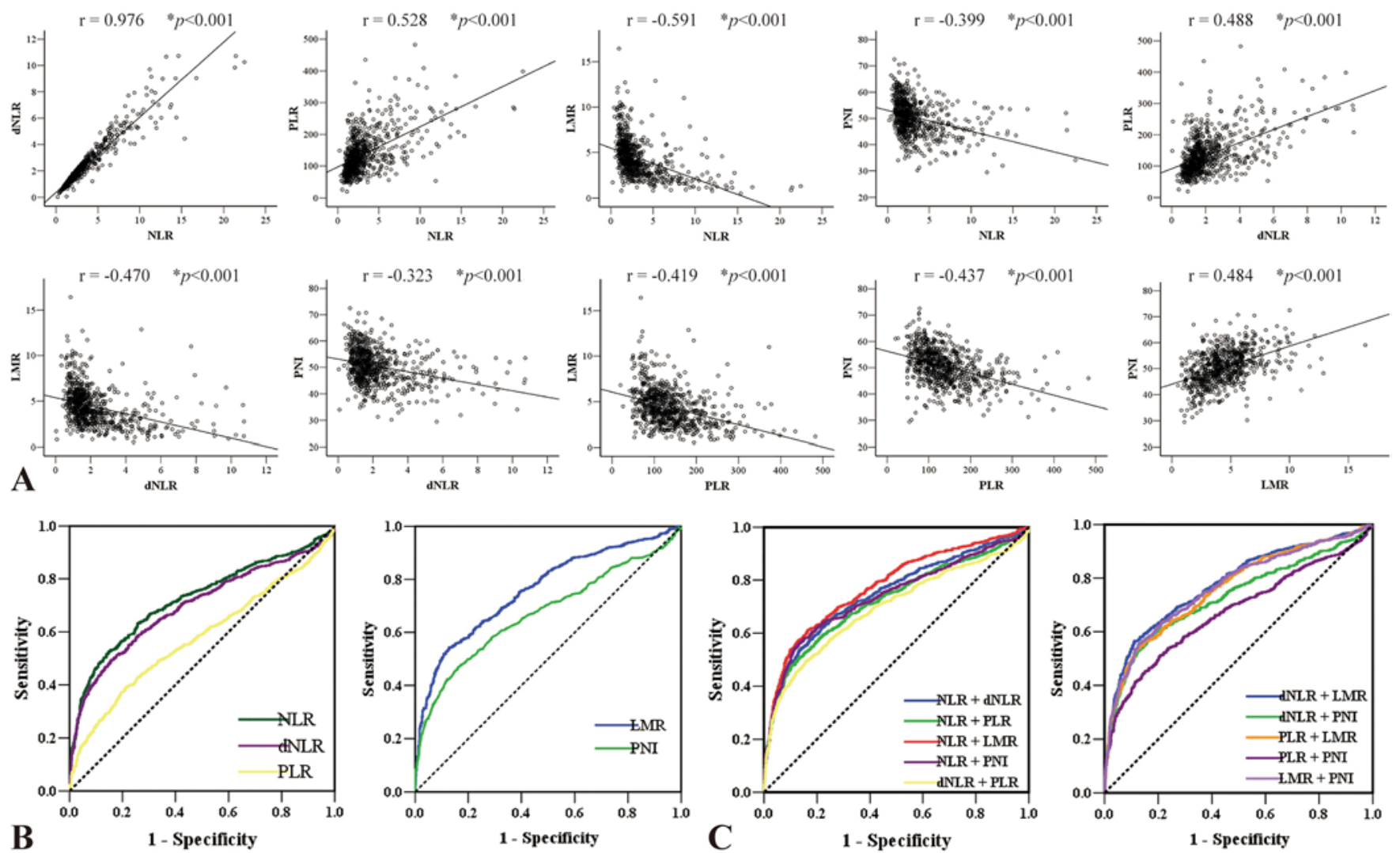

FIG. 3. The diagnostic value of preoperative inflammatory markers in patients with glioma. A: Correlations between NLR, dNLR, PLR, LMR, and PNI in patients with glioma. B: Diagnostic efficacy of NLR, dNLR, PLR, LMR, and PNI in patients with glioma. C: Diagnostic efficacy of paired combinations of NLR, dNLR, PLR, LMR, and PNI in patients with glioma. Figure is available in color online only. 
TABLE 3. Diagnostic value of NLR, dNLR, PLR, LMR, PNI, and their combinations

\begin{tabular}{llll}
\hline & & AUC $(95 \%$ Cl $)$ & \\
\cline { 2 - 4 } Marker & Glioma vs Others & GBM vs Others & GBM vs Grades I-III \\
\hline NLR & $0.722(0.697-0.747)$ & $0.860(0.832-0.887)$ & $0.811(0.778-0.844)$ \\
\hline dNLR & $0.696(0.670-0.722)$ & $0.840(0.810-0.869)$ & $0.797(0.763-0.832)$ \\
\hline PLR & $0.576(0.549-0.604)$ & $0.678(0.641-0.715)$ & $0.662(0.622-0.702)$ \\
\hline LMR & $0.760(0.738-0.783)$ & $0.837(0.811-0.863)$ & $0.743(0.707-0.779)$ \\
\hline PNI & $0.672(0.646-0.698)$ & $0.740(0.706-0.773)$ & $0.661(0.622-0.701)$ \\
\hline NLR+dNLR & $0.748(0.724-0.771)$ & $0.869(0.843-0.895)$ & $0.815(0.782-0.848)$ \\
\hline NLR+PLR & $0.723(0.698-0.748)$ & $0.860(0.833-0.887)$ & $0.811(0.778-0.844)$ \\
\hline NLR+LMR & $0.777(0.755-0.799)$ & $0.882(0.859-0.905)$ & $0.82(0.788-0.852)$ \\
\hline NLR+PNI & $0.739(0.714-0.763)$ & $0.865(0.840-0.891)$ & $0.814(0.782-0.846)$ \\
\hline$d N L R+P L R$ & $0.696(0.670-0.721)$ & $0.838(0.809-0.868)$ & $0.796(0.761-0.830)$ \\
\hline$d N L R+L M R$ & $0.778(0.755-0.800)$ & $0.881(0.858-0.905)$ & $0.819(0.787-0.852)$ \\
\hline$d N L R+P N I$ & $0.728(0.704-0.753)$ & $0.855(0.829-0.881)$ & $0.803(0.770-0.836)$ \\
\hline PLR+LMR & $0.760(0.737-0.783)$ & $0.841(0.815-0.867)$ & $0.755(0.719-0.791)$ \\
\hline PLR+PNI & $0.673(0.647-0.699)$ & $0.757(0.724-0.790)$ & $0.696(0.657-0.735)$ \\
\hline LMR+PNI & $0.765(0.742-0.788)$ & $0.845(0.820-0.870)$ & $0.749(0.714-0.785)$ \\
\hline
\end{tabular}

Others include healthy controls and patients with nonlesional epilepsy, acoustic neuroma, and meningioma.

GBM. In this study, we assessed the levels of several preoperative inflammatory markers in glioma patients, including NLR, dNLR, PLR, LMR, and PNI, explored their correlations with tumor grade, and evaluated their diagnostic value for glioma, particularly for GBM.

Recently, preoperative NLR has been recognized as a prognostic factor in GBM. Elevated NLR has been demonstrated to be correlated with poorer survival and a worse prognosis in GBM patients. ${ }^{3,12}$ Except in cases of GBM at first progression, an NLR $>4$ prior to a second surgery is also a poor prognostic factor in GBM. ${ }^{20}$ However, apart from the value of NLR in predicting survival and prognosis in GBM, the levels of NLR and dNLR in glioma and their correlations with tumor grade are still unknown. In the present study, we provided first evidence that patients with glioma have higher levels of preoperative NLR and dNLR than patients with nonlesional epilepsy, acoustic neuroma, or meningioma and healthy controls. Specifically, it is possible for NLR and dNLR to differentiate glioma patients from patients with nonlesional epilepsy, acoustic neuroma, or meningioma and healthy individuals. According to our data, the increases in preoperative NLR and dNLR in glioma patients result from an elevation in the neutrophil count and a relative reduction in the lymphocyte count, suggesting heightened neutrophil-dependent inflammatory reactions and a decreased lymphocyte-mediated antitumor immune response. Unfortunately, the mechanisms underlying the increase in neutrophils and the decrease in lymphocytes are not clearly defined. Some authors ascribe the stimulation of neutrophils' infiltration to tumor-secreted neutrophil chemotactic factors and reactive oxygen species generated by tumor cells. ${ }^{8,31}$ In addition, GBM tumor cells have been shown to reduce lymphocyte infiltration by secreting immunosuppressive cytokines such as interleukin-10 (IL-10), IL-2, and tumor growth factor- $\beta$ (TGF- $\beta$ ). ${ }^{2}$ Additionally, we also observed a positive correlation between NLR, dNLR, and tumor grade in our study. Consis- tent with our results, a pilot study by Zadora et al. proved preoperative NLR to be a predictive factor for WHO grading of glioma. ${ }^{31}$ Liang and colleagues reported that an increase in neutrophil infiltration into tumors is significantly correlated with glioma grade, which could partly explain the positive correlations between NLR, dNLR, and tumor grade..$^{18}$

In previous studies, PLR has been demonstrated to be a prognostic factor for various cancers. ${ }^{4,16}$ In a study of GBM, Han et al. reported that an increased level of PLR was correlated with poorer survival even though the prognostic significance of PLR was far less than that of NLR..$^{12}$ Here, we initially reported a higher level of preoperative PLR in patients with glioma compared with healthy controls and nonlesional epilepsy patients, whereas no significant differences were found between glioma patients and patients with acoustic neuroma or meningioma. This finding suggests that PLR may not have the ability to differentiate glioma from other intracranial space-occupying lesions such as acoustic neuroma and meningioma. We also revealed a positive correlation between PLR and tumor grade. Relatively high levels of PLR were found in glioma patients. However, the underlying mechanism for the elevation of PLR in glioma and GBM needs further investigation.

As for LMR, its decreased level has been identified as a poor prognostic index for various cancers such as urothelial carcinoma, renal cell carcinoma, and soft tissue sarcoma. ${ }^{9,26}$ Moreover, recent studies have revealed that LMR is associated with pathological T (pT) stage in esophageal cancer, TNM stage in gastric cancer, and Fuhrman grade in renal cell carcinoma. ${ }^{9}$ However, little is known about the levels of LMR in glioma. Here, we demonstrated that the level of preoperative LMR is significantly lower in patients with glioma than in healthy controls and is inversely correlated with glioma grade. Our data suggest that decreased LMR is reflected by a reduction in lymphocyte counts and 
GBM vs Others

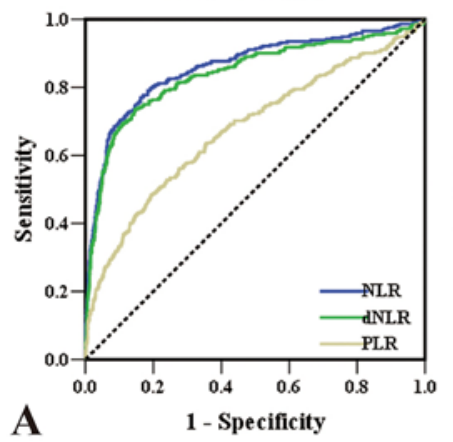

GBM vs Others

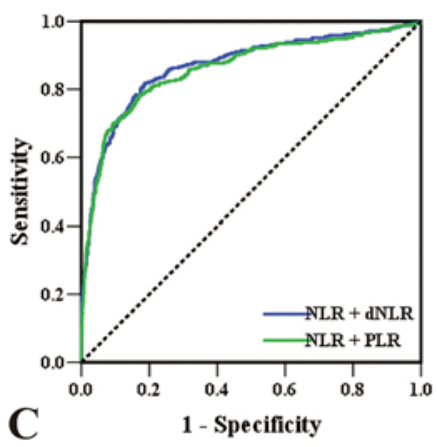

GBM vs Grade I, II, III

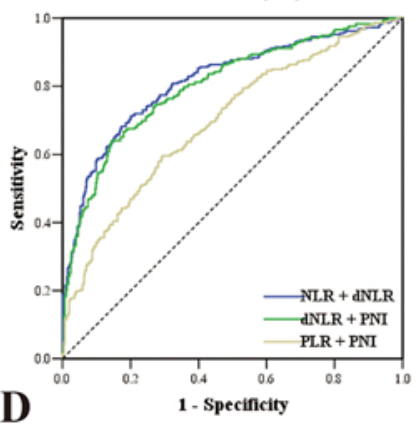

GBM vs Others

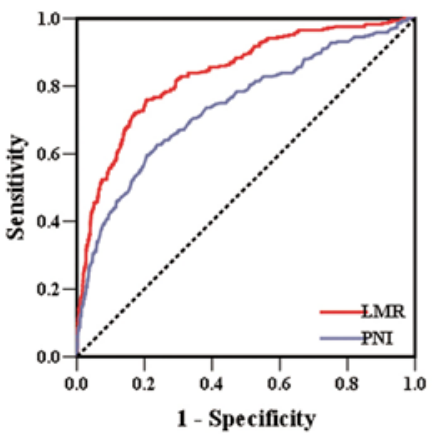

GBM vs Others

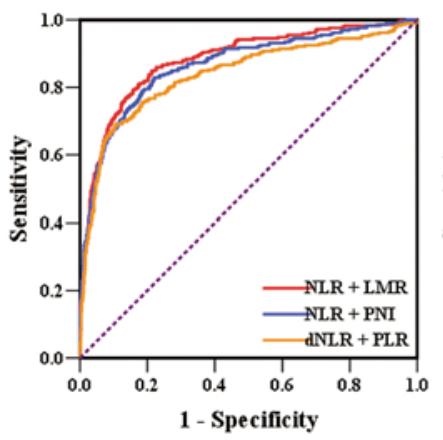

GBM vs Grade I, II, III

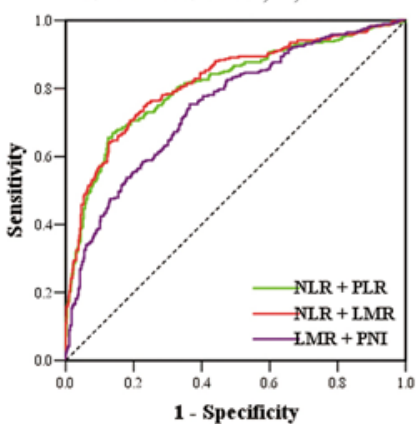

GBM vs Grade I, II, III

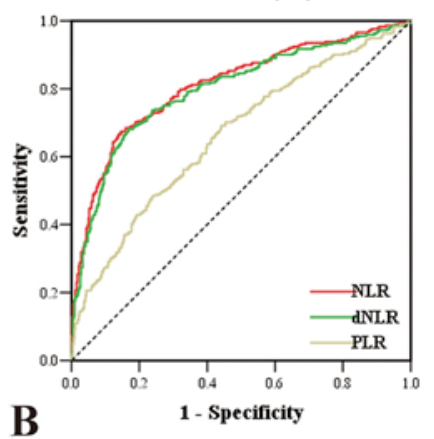

GBM vs Others

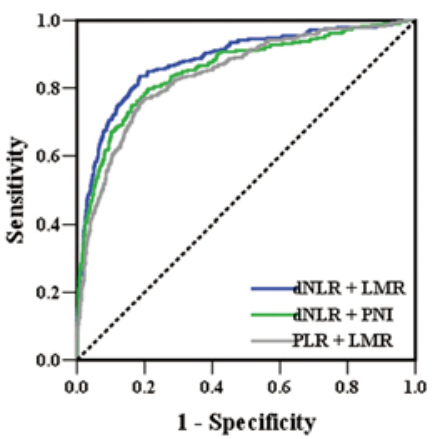

GBM vs Grade I, II, III

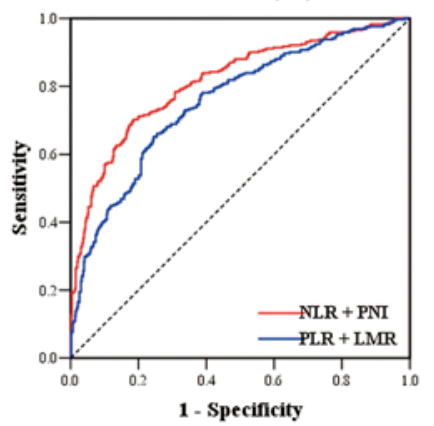

GBM vs Grade I, II, III

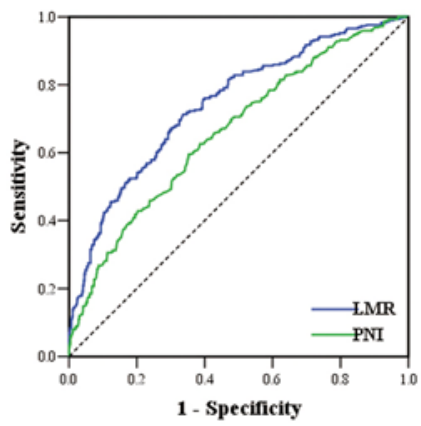

GBM vs Others

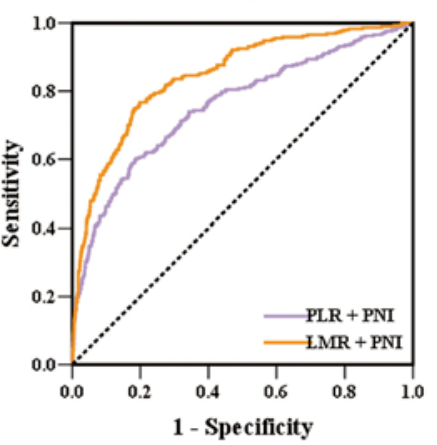

GBM vs Grade I, II, III

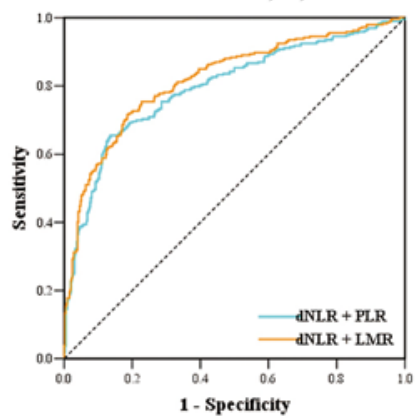

FIG. 4. Diagnostic value of preoperative inflammatory markers in patients with GBM. The diagnostic value of NLR, dNLR, PLR, LMR, and PNI was evaluated by ROC analysis in patients with GBM tested against patients with nonlesional epilepsy, acoustic neuroma, meningioma, and healthy controls (A) or against patients with glioma Grades I-III (B). Diagnostic efficacy for paired combinations of NLR, dNLR, PLR, LMR, and PNI was assessed in patients with GBM tested against patients with nonlesional epilepsy, acoustic neuroma, meningioma, and healthy controls (C) or against patients with glioma Grades I-III (D). Figure is available in color online only.

an elevation of monocyte counts in patients suffering from glioma. The reason for high infiltration of macrophages in glioma remains speculative at present. Macrophages can be recruited and induced to become M2 phenotypes by a wide variety of factors secreted by glioma cells, including IL-10, IL-4, IL-6, macrophage-colony stimulating factor (M-CSF), TGF- $\beta$, prostaglandin E2 (PGE2), and periostin. ${ }^{27,32}$ Importantly, the level of LMR was lower in patients with glioma than in patients with nonlesional epilepsy, acoustic neuroma, or meningioma, indicating that LMR may be a useful biomarker for the differential diagnosis of glioma versus other intracranial diseases. We also noticed a significantly lower level of LMR in patients with Grade I glioma compared to that in healthy controls, indicating that LMR may be useful for the early detection of glioma.
Importantly, preoperative serum albumin and PNI levels were decreased in glioma patients relative to levels in the groups with healthy controls, nonlesional epilepsy, acoustic neuroma, and meningioma. Serum albumin and PNI are widely used as parameters for nutritional status and systemic inflammatory response, and low levels of them have been reported to be associated with poor survival in several types of cancer. ${ }^{710}$ A recent publication by Han et al. ${ }^{11}$ revealed that GBM patients with lower preoperative serum albumin levels have worse survival. Additionally, our data revealed a negative correlation between PNI and tumor grade, with the lowest values in patients with GBM.

Clinically, CT and MRI are the diagnostic imaging modalities of choice when glioma is suspected, while histological examination of the tumor tissue remains the cur- 
rent gold standard. ${ }^{13}$ However, these diagnostic approaches have their own limitations. Histological examination is invasive and expensive because of the resection or stereotactic biopsy procedure. ${ }^{23}$ Moreover, the general application of CT and MRI as routine screening tools is limited due to the high cost. Unfortunately, unlike for other cancers, no circulating serum or plasma biomarkers have been established for the routine clinical management of patients with glioma. ${ }^{17,30}$ Here, we assessed the performance of preoperative NLR, dNLR, PLR, LMR, and PNI for glioma diagnosis using ROC curve analysis. For single factors, LMR is superior to the other 4 markers as a diagnostic factor for glioma and may also be used as a potential biomarker for early detection of glioma. For paired combinations of these 5 markers, the best diagnostic performance was proved in the use of preoperative NLR+LMR and dNLR+LMR. Routine blood tests and hepatic function tests are noninvasive, low cost, and widely available for disease screening. A 2-stage approach may be useful for screening glioma patients, with patients positive for the NLR+LMR or dNLR+LMR combination test referred for neuroimaging.

The highest median values of NLR, dNLR, and PLR, as well as the lowest median values of LMR and PNI, in GBM patients in this study provide a basis for proposing these 5 markers as diagnostic biomarkers of GBM. Interestingly, NLR demonstrated the greatest accuracy in predicting GBM compared with either healthy controls plus patients with other intracranial diseases or patients suffering from glioma Grades I-III. Similarly, among the paired combinations, NLR+LMR offered the best diagnostic performance for GBM. Moreover, the NLR+LMR combination may be a useful biomarker for GBM diagnosis and the differential diagnosis of GBM versus acoustic neuroma or meningioma or even low-grade glioma. Currently, neuroimaging studies are not reliable in distinguishing features of tumor grade.,13 It has been reported that even advanced MRI provides diagnostic specificity ranging from only $50 \%$ to $80 \%$ for distinguishing GBM from low-grade glioma, lymphoma, metastases, abscesses, and subacute infarcts. ${ }^{13}$ Therefore, the preoperative NLR+LMR combination has important accessory diagnostic value for the differential diagnosis of patients with GBM and other lowgrade gliomas.

There are a few limitations to our study. First, our cohort consisted of a relatively small proportion of patients with glioma; in particular, only a limited number of Grade I patients were included. Although we retrospectively acquired data from multiple centers to minimize per definition the selection bias, larger studies are needed to confirm our preliminary results. Second, the high NLR, dNLR, and PLR, as well as the low LMR and PNI, observed in our study may reflect a nonspecific inflammatory response due to glioma; that is, any inflammatory or malignant process can result in the changes of these parameters. Hence, there is a risk of generating false-positive test results in screening asymptomatic populations.

Apart from these limitations, we consider our findings to be clinically important in 3 respects: 1) to provide data on potential biomarkers for the screening and diagnosis of glioma. Despite a possible false-positive test, application of the NLR+LMR combination could lead to the referral of more individuals with characteristic or uncharacteristic symptoms to CT or MRI, with the subsequent diagnosis of more patients with glioma and a potential increase in the number of patients operated on and possibly cured. 2) The utility of NLR+LMR, especially when combined with neuroimaging tests, may help to distinguish glioma from acoustic neuroma and meningioma as well as GBM from low-grade glioma. 3) The biomarkers could provide useful information for the therapeutic plan for glioma. For example, NLR was significantly elevated in advanced glioma; thus, the use of drugs acting against neutrophil-derived factors may be a choice for patients with advanced glioma.

\section{Conclusions}

In this multicenter study, we provide the first evidence that patients with glioma have higher levels of NLR, dNLR, and PLR and lower levels of LMR and PNI than patients with nonlesional epilepsy, acoustic neuroma, or meningioma and healthy controls. Values for NLR, dNLR, and PLR are positively correlated with glioma grade, whereas LMR and PNI values are negatively correlated with glioma grade. We also demonstrate that the NLR+LMR combination as a noninvasive biomarker has relatively high sensitivity and specificity for glioma diagnosis, the differential diagnosis of glioma from acoustic neuroma and meningioma, GBM diagnosis, and the differential diagnosis of GBM from low-grade glioma. The combination of NLR+LMR may be applied as a routine screening tool for patients with glioma.

\section{Acknowledgments}

We thank the biomedical laboratory scientists for excellent technical assistance with handling the blood samples. We also thank the patients for their participation in this study.

\section{References}

1. Ahmed R, Oborski MJ, Hwang M, Lieberman FS, Mountz JM: Malignant gliomas: current perspectives in diagnosis, treatment, and early response assessment using advanced quantitative imaging methods. Cancer Manag Res 6:149170, 2014

2. Baecher-Allan C, Anderson DE: Regulatory cells and human cancer. Semin Cancer Biol 16:98-105, 2006

3. Bambury RM, Teo MY, Power DG, Yusuf A, Murray S, Battley JE, et al: The association of pre-treatment neutrophil to lymphocyte ratio with overall survival in patients with glioblastoma multiforme. J Neurooncol 114:149-154, 2013

4. Cummings M, Merone L, Keeble C, Burland L, Grzelinski M, Sutton K, et al: Preoperative neutrophil:lymphocyte and platelet:lymphocyte ratios predict endometrial cancer survival. Br J Cancer 113:311-320, 2015

5. Deng Q, He B, Liu X, Yue J, Ying H, Pan Y, et al: Prognostic value of pre-operative inflammatory response biomarkers in gastric cancer patients and the construction of a predictive model. J Transl Med 13:66, 2015

6. Gasparyan AY, Ayvazyan L, Mikhailidis DP, Kitas GD: Mean platelet volume: a link between thrombosis and inflammation? Curr Pharm Des 17:47-58, 2011

7. Geng Y, Qi Q, Sun M, Chen H, Wang P, Chen Z: Prognostic nutritional index predicts survival and correlates with systemic inflammatory response in advanced pancreatic cancer. Eur J Surg Oncol 41:1508-1514, 2015 
8. Gregory AD, Houghton AM: Tumor-associated neutrophils: new targets for cancer therapy. Cancer Res 71:2411-2416, 2011

9. Gu L, Li H, Chen L, Ma X, Li X, Gao Y, et al: Prognostic role of lymphocyte to monocyte ratio for patients with cancer: evidence from a systematic review and meta-analysis. Oncotarget 7:31926-31942, 2016

10. Gupta D, Lis CG: Pretreatment serum albumin as a predictor of cancer survival: a systematic review of the epidemiological literature. Nutr J 9:69, 2010

11. Han S, Huang Y, Li Z, Hou H, Wu A: The prognostic role of preoperative serum albumin levels in glioblastoma patients. BMC Cancer 15:108, 2015

12. Han S, Liu Y, Li Q, Li Z, Hou H, Wu A: Pre-treatment neutrophil-to-lymphocyte ratio is associated with neutrophil and T-cell infiltration and predicts clinical outcome in patients with glioblastoma. BMC Cancer 15:617, 2015

13. Hochberg FH, Atai NA, Gonda D, Hughes MS, Mawejje B, Balaj L, et al: Glioma diagnostics and biomarkers: an ongoing challenge in the field of medicine and science. Expert Rev Mol Diagn 14:439-452, 2014

14. Jia J, Zheng X, Chen Y, Wang L, Lin L, Ye X, et al: Stagedependent changes of preoperative neutrophil to lymphocyte ratio and platelet to lymphocyte ratio in colorectal cancer. Tumour Biol 36:9319-9325, 2015

15. Kilincalp S, Çoban Ş, Akinci H, Hamamcı M, Karaahmet F, Coşkun Y, et al: Neutrophil/lymphocyte ratio, platelet/ lymphocyte ratio, and mean platelet volume as potential biomarkers for early detection and monitoring of colorectal adenocarcinoma. Eur J Cancer Prev 24:328-333, 2015

16. Koh CH, Bhoo-Pathy N, Ng KL, Jabir RS, Tan GH, See MH, et al: Utility of pre-treatment neutrophil-lymphocyte ratio and platelet-lymphocyte ratio as prognostic factors in breast cancer. Br J Cancer 113:150-158, 2015

17. Kros JM, Mustafa DM, Dekker LJ, Sillevis Smitt PA, Luider TM, Zheng PP: Circulating glioma biomarkers. Neuro Oncol 17:343-360, 2015

18. Liang J, Piao Y, Holmes L, Fuller GN, Henry V, Tiao N, et al: Neutrophils promote the malignant glioma phenotype through S100A4. Clin Cancer Res 20:187-198, 2014

19. McMillan DC: Systemic inflammation, nutritional status and survival in patients with cancer. Curr Opin Clin Nutr Metab Care 12:223-226, 2009

20. McNamara MG, Lwin Z, Jiang H, Templeton AJ, Zadeh G, Bernstein M, et al: Factors impacting survival following second surgery in patients with glioblastoma in the temozolomide treatment era, incorporating neutrophil/lymphocyte ratio and time to first progression. J Neurooncol 117:147-152, 2014

21. Omuro A, DeAngelis LM: Glioblastoma and other malignant gliomas: a clinical review. JAMA 310:1842-1850, 2013

22. Ostrom QT, Gittleman H, Stetson L, Virk SM, BarnholtzSloan JS: Epidemiology of gliomas. Cancer Treat Res 163:1-14, 2015

23. Qu S, Guan J, Liu Y: Identification of microRNAs as novel biomarkers for glioma detection: a meta-analysis based on 11 articles. J Neurol Sci 348:181-187, 2015

24. Raffetti E, Donato F, Castelnuovo F, Ladisa N, Paraninfo G,
Di Filippo E, et al: The prognostic role of systemic inflammatory markers on HIV-infected patients with non-Hodgkin lymphoma, a multicenter cohort study. J Transl Med 13:89, 2015

25. Sowers JL, Johnson KM, Conrad C, Patterson JT, Sowers LC: The role of inflammation in brain cancer. Adv Exp Med Biol 816:75-105, 2014

26. Szkandera J, Gerger A, Liegl-Atzwanger B, Absenger G, Stotz M, Friesenbichler J, et al: The lymphocyte/monocyte ratio predicts poor clinical outcome and improves the predictive accuracy in patients with soft tissue sarcomas. Int J Cancer 135:362-370, 2014

27. Wei J, Gabrusiewicz K, Heimberger A: The controversial role of microglia in malignant gliomas. Clin Dev Immunol 2013:285246, 2013

28. Weller M, van den Bent M, Hopkins K, Tonn JC, Stupp R, Falini A, et al: EANO guideline for the diagnosis and treatment of anaplastic gliomas and glioblastoma. Lancet Oncol 15:e395-e403, 2014

29. Yang C, Wang C, Chen X, Chen S, Zhang Y, Zhi F, et al: Identification of seven serum microRNAs from a genomewide serum microRNA expression profile as potential noninvasive biomarkers for malignant astrocytomas. Int J Cancer 132:116-127, 2013

30. Yue X, Lan F, Hu M, Pan Q, Wang Q, Wang J: Downregulation of serum microRNA-205 as a potential diagnostic and prognostic biomarker for human glioma. J Neurosurg 124:122-128, 2016

31. Zadora P, Dabrowski W, Czarko K, Smolen A, KotlinskaHasiec E, Wiorkowski K, et al: Preoperative neutrophil-lymphocyte count ratio helps predict the grade of glial tumor-a pilot study. Neurol Neurochir Pol 49:41-44, 2015

32. Zhou W, Ke SQ, Huang Z, Flavahan W, Fang X, Paul J, et al: Periostin secreted by glioblastoma stem cells recruits M2 tumour-associated macrophages and promotes malignant growth. Nat Cell Biol 17:170-182, 2015

\section{Disclosures}

The authors report no conflict of interest concerning the materials or methods used in this study or the findings specified in this paper.

\section{Author Contributions}

Conception and design: S Huang, Zheng. Acquisition of data: Zheng, J Huang, Wang. Analysis and interpretation of data: Zheng. Drafting the article: Zheng, Ou. Critically revising the article: J Huang. Reviewed submitted version of manuscript: Zheng. Statistical analysis: Zheng, J Huang. Study supervision: S Huang, Ou.

\section{Correspondence}

Sheng-yue Huang, Department of Neurosurgery, Fujian Provincial Hospital, No. 143 East St., Fuzhou, Fujian Province 350001, China.email: hsysjwk@sina.com. 